\title{
New Developments and Applications of High Pressure Freezing
}

\author{
Kent McDonald
}

Electron Microscope Lab, University of California, Berkeley, CA 94720-3330

High pressure freezing (HPF) is one of the most important specimen preparation advances for biological electron microscopy since the development of glutaraldehyde fixation [1]. HPF provides superior cellular preservation for morphological studies and better antigen retention for immunolabeling work than most conventional, room temperature methods (Figures 1-4). One powerful advantage of ultrarapid freezing is that it is extremely fast compared to conventional chemical fixation methods. Cells are immobilized in milliseconds instead of the seconds to minutes it takes fixatives like glutaraldehyde to diffuse into cells. Fast freezing is also non-selective, i.e., all chemical species are arrested equally whereas chemical fixative cross-linking is selective.

Despite obvious superiority over conventional methods, HPF has not emerged as a routine method for EM specimen preparation. Although the relatively high cost of the machines has had something to do with that, it is also because there have been few applications that absolutely required HPF. Now that is beginning to change as EM studies contribute to the effort to characterize cellular substructure down to the atomic level of detail [2].

What are the applications and uses for HPF? First and foremost, it is a technique for preserving high resolution ultrastructural information in biological samples. This makes it ideal, if not essential, for sectioning and tomography of frozen hydrated material $[3,4]$ as well as resin-embedded material [57; see also abstract of Müller-Reichert \& O'Toole, this symposium]. But it is also emerging as an essential fixation technique for EM studies of the important model organisms such as Drosophila, $C$. elegans, E. coli, S. cerevisiae, and Arabidopsis among others. These are organisms that are difficult to fix well by conventional methods and HPF facilitates accurate phenotype characterization as well as EM immunolocalization studies. In this presentation we will cover these applications of HPF as well as consider the types of high pressure freezing machines, how they work, and how to optimize their use. We will discuss artefacts of HPF and some of the processing problems that are encountered following HPF.

\section{References}

[1] Sabatini, D.D., Bensch, K., and R.J. Barnett. 1963. J. Cell. Biol. 17:19-58.

[2] Sali, A., R. Glaeser, T. Earnest \& W. Baumeister. 2003 Nature 422:216-225.

[3] Hsieh, C.-E., M. Marko, J. Frank \& C.A. Mannella. 2002. J. Struct. Biol. 138:63-73.

[4] Al-Amoudi, A., J.-J. Chang, A. Leforestier, A. McDowall, L.M. Salamin, L.P.O. Norlén, K.

Richter, N. Sartori Blanc, D. Studer, and J. Dubochet. 2004. EMBO Journal 2004:1-8.

[5] McEwen, B.F., and M. Marko. 2001.. J. Histoch. Cytoch. 49:553-563.

[6] McIntosh, J.R. 2001. J. Cell. Biol. 153:F25-F32

[7] O’Toole, E. T., K. L. McDonald, J. Mäntler, J.R. McIntosh, A.A. Hyman \& T. Müller-Reichert. 2003. J. Cell Biology 163:451-456. 

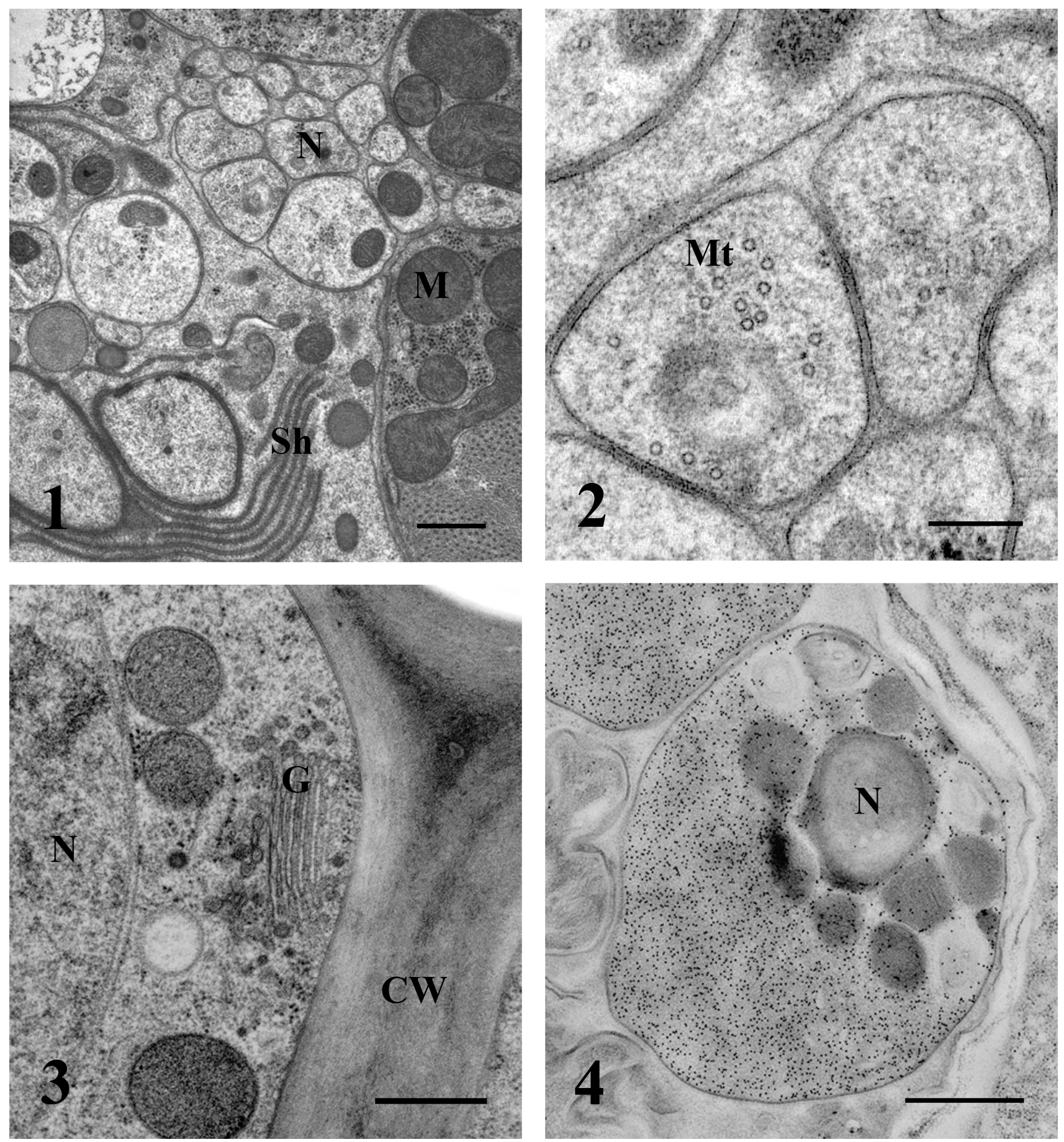

Figure 1. General cytoplasmic structure in the head region of $C$. elegans showing good preservation of a nerve bundle $(\mathrm{N})$, mitochonria $(\mathrm{M})$ and sheath membranes $(\mathrm{Sh})$. Bar $=0.5 \mu \mathrm{m}$.

Figure 2. Detail of Fig. 1 showing microtubules (Mt) in a nerve bundle.. Bar $=200 \mathrm{~nm}$.

Figure 3. Cytoplasmic preservation in the plant Pittosporum undulatum showing good ultrastructure of the nucleus $(\mathrm{N})$, Golgi apparatus $(\mathrm{G})$ and the cell wall $(\mathrm{CW})$. Bar $=0.5 \mu \mathrm{m}$.

Figure 4. Immunolabeling of major sperm protein (MSP) with $10 \mathrm{~nm}$ gold in sperm cells of $C$. elegans. Note absence of labeling in the condensed chromatin (N). Antibody against MSP from David Greenstein, Vanderbilt University. Bar $=0.5 \mu \mathrm{m}$. 